\title{
Stent Boost Subtract Imaging for the Assessment of Optimal Stent Deployment in Coronary Ostial Lesion Intervention
}

\section{Comparison With Intravascular Ultrasound}

\author{
Jiao ZhanG, ${ }^{1}$ MD, YuanYuan DuAn, ${ }^{2}$ MD, ZhiGeng Jin, ${ }^{3}$ MD, YuJie WeI, ${ }^{3}$ MD, ShengLi YANG, ${ }^{3}$ MD, \\ JianPing Luo, ${ }^{3} \mathrm{MD}$, DongXing MA, ${ }^{3} \mathrm{MD}$, LiMin JinG, ${ }^{3} \mathrm{MD}$, and HuiLiang LiU, ${ }^{3} \mathrm{MD}$
}

\begin{abstract}
SUMMARY
Percutaneous coronary intervention (PCI) of ostial lesions is complex and is technically very demanding. Intravascular ultrasound (IVUS) is considered the gold standard method to guide PCI but has several limitations. Stent boost subtract (SBS) imaging is an enhancement of the radiologic edge of the stent by digital management of regular X-ray images. The purpose of this study was to determine the availability of stent enhancement with SBS during ostial PCI by comparison with IVUS.

We investigated SBS and IVUS after stent implantation in 58 ostial lesions in 55 patients. SBS and IVUS were performed in all patients to obtain improved stent location and to detect optimal release and deployment. We defined the SBS and IVUS criteria for accuracy of stent location and adequate stent deployment. IVUS findings showed that stent location was generally good. The location was accurate in $48(82.8 \%)$ and inadequate stent deployment was observed in 10 of $58(17.2 \%)$. Eight SBS images showed inadequate stent expansion. SBS predicted inadequate findings of IVUS with $100 \%$ specificity and $80 \%$ sensitivity, while a significant positive correlation was observed between SBS-MSA and MSA by IVUS with a regression coefficient of 0.95 .

Imaging techniques have a primary role during ostial PCI. SBS is a simple and quick method that offers several advantages, enabling improved stent location, adequate stent expansion, and optimal apposition of the struts to the wall. SBS imaging could be conventionally used during ostial PCI, especially in centers where IVUS is not used routinely. (Int Heart J 2015; 56: 37-42)
\end{abstract}

Key words: Percutaneous coronary intervention, Apposition, Stent location, Stent expansion

$\mathrm{P}$ ercutaneous coronary intervention (PCI) of coronary ostial lesions is technically difficult leading to higher rates of adverse cardiac events compared with PCI of non-ostial coronary lesions. ${ }^{1-4)}$ The challenges of PCI of an ostial lesion may depend not only upon the lesion location (ie, aorto-ostial versus non-aorto-ostial), but also on the plaque burden and lesion morphology. Ostial lesions are less compliant compared with non-ostial lesions due to increased fibrosis, calcification, and muscular/elastic tissue, the latter of which is especially evident in aorto-ostial disease. ${ }^{5)}$ Complete stent coverage of ostial lesions can be problematic, with inaccurate stent placement leading to higher rates of restenosis. ${ }^{6}$ Compared with coronary angiography, intravascular ultrasound (IVUS) allows for a more detailed assessment of coronary anatomy, including plaque characteristics and morphology, vessel size, and precise localization of the true ostium. ${ }^{7)}$ The only reliable way to exclude insufficient stent deployment with certainty is IVUS. ${ }^{8-10)}$ However, in daily practice, this technique is not used routinely because it is expensive, time-consuming, and there is a learning curve (requiring trained operators and laboratory staff). ${ }^{11)}$ Moreover, it is difficult to obtain an accurate location of the stent before the stent is released. Stent boost subtract (SBS) is a recently developed imaging technique that enhances stent fluoroscopic visibility. Through motion-corrected acquisition frames, an enhanced picture of the stent and its relation with the vessel wall is obtained. The purpose of this study was to evaluate SBS imaging during ostial lesion PCI, using IVUS as an anatomical standard for adequate stent deployment.

\section{Methods}

Patient population: From March 2011 to Dec 2013, 467 patients were screened for participation. This single-centre prospective study finally included 55 patients who underwent ostial lesion angioplasty with the use of SBS and IVUS. Inclusion criteria were patients with de novo coronary ostial lesions defined as a lesion occurring within $3 \mathrm{~mm}$ of the os-

From the ${ }^{1}$ Chinese People Liberation Army General Hospital, Departments of ${ }^{2}$ South and ${ }^{3}$ Cardiology, the General Hospital of Chinese People's Armed Police Forces, Beijing, China.

Address for correspondence: Huiliang Liu, MD, Department of Cardiology, General Hospital of Chinese People's Armed Police Forces, No.69, Yongding Road, Haidian District, Beijing 100039, China. E-mail: liuhuiliang1961@163.com

Received for publication May 26, 2014. Revised and accepted June 23, 2014.

Released in advance online on J-STAGE December 24, 2014.

All rights reserved by the International Heart Journal Association. 
tium of a native aorto-ostial (ie, left main or right coronary arteries) or major coronary vessel (left anterior descending, left circumflex, and ramus intermedius arteries) without any extension proximally or distally into an adjacent branch vessel and clinical indication for PCI with stent implantation. Exclusion criteria included any contraindication for PCI or PCI without stent implantation. The study protocol was approved by the ethics committees of the General Hospital of Chinese People's Armed Police Forces on January 8, 2011 and all patients signed informed written consent forms.

Procedures: Baseline characteristics, symptomatic status, number of diseased vessels per patient, and ostial lesions were assessed. The procedure was performed by one of three experienced interventionalists. All decisions regarding material, device selection, or the appropriate treatment strategy were left to the attending physician. In addition, the operator was blind to the data collection. Measurements were repeated off-line by two independent observers. All reported SBS and IVUS parameters required the agreement of 2 observers (DYY and JZG) who were blinded to the clinical and procedural characteristics.

Stent boost subtract imaging: Angiographic images were obtained using an Allura Xper FD 20/10 system (Philips Medical Systems, Best, The Netherlands). SBS images were obtained with the deflated balloon still in place, after stent implantation. The balloon markers were used as reference points. After stent deployment and intracoronary nitroglycerin administration, 3to 4-second motion-corrected runs were acquired. The use of a contrast-filled subtraction image was at the discretion of the operator. Post-acquisition, stent location was verified and balloon marks were manually corrected if necessary. The minimum diameters of the stent and stent edge diameters as the reference of the stent were determined automatically using the SBS software, and the symmetrical index of the stent was calculated as the minimum diameter/maximum diameter. Each of the SBS images was evaluated from two orthogonal directions. Minimum stent area (MSA) was calculated from stent diameter of two orthogonal directions by assuming it to be an oval (SB-MSA). Criteria for accuracy of stent location and adequate stent expansion by SB imaging are shown in Table I.

Intravascular ultrasound: IVUS was performed using the standard technique. IVUS was performed either pre-intervention or post-intervention. One commercially available system (Eagle Eye, Volcano Therapeutics, Inc., Rancho Cordova, CA, USA) was used. IVUS images were recorded after administra- tion of $100-200 \mathrm{mg}$ of nitroglycerin. The ultrasound catheter was advanced $5 \mathrm{~mm}$ beyond the lesion/stent and was pulled back to a point $5 \mathrm{~mm}$ proximal to the lesion/stent using motorized transducer pull-back at $0.5 \mathrm{~mm} / \mathrm{s}$. IVUS was performed and interpreted by the treating physician and at least one experienced IVUS technician. Minimum stent area and the lumen for the proximal and distal stent edge as the reference area (RA) were measured. The symmetry index by IVUS was defined as the minimum stent diameter/maximum stent diameter at the most elliptic part of the stent. The presence of edge dissection, hematoma at the stent edge, and incomplete apposition of the stent over its entire length were assessed visually. Criteria for accuracy of stent location and adequate stent expansion by IVUS are described in Table I.

Statistical analysis: Data are presented as the mean \pm SD for continuous variables. Linear regression analysis was performed to evaluate the correlation between SBS-MSA and MSA by IVUS. The resulting sensitivities and specificities were calculated. All statistical analyses were performed using SPSS version 22.0 (SPSS Inc., Chicago, IL, USA), and a $P$ value of $<0.05$ was considered statistically significant.

\section{Results}

From March 2011 to December 2013, 467 patients were screened for participation. Of these, only 55 patients were enrolled in the study. The causes for exclusion were PCI for bifurcation lesions $(n=324)$, no check of IVUS $(n=67)$, coronary artery bypass grafting after angiography $(n=54)$, occurrence of PCI complications $(n=12)$, only thrombus aspiration $(n=6)$, poor SBS image quality $(n=3)$, and poor IVUS image quality $(n=1)$ (Figure 1). Baseline patient and ostial lesions characteristics are described in Table II. The mean age was $65.4 \pm 13.9$ years and $36 \%$ were male. Clinical presentation was an ST elevation acute myocardial infarction in 3 patients $(5 \%)$, non-ST elevation acute coronary syndrome in 11 patients $(20 \%)$, unstable angina in 34 patients $(62 \%)$, and stable angina in 7 patients (13\%). A total of 58 ostial lesions were treated with stent implantation and evaluated with SBS and IVUS during the procedure.

The ostial lesion characteristics and stenting techniques of the 58 distinct coronary ostial lesions are shown in Table III. The predominant ostial vessel location was in the left anterior descending coronary artery followed by the right coronary ar-

Table I. Definitions of Adequate Stent Expansion and Accuracy of Stent Location

\begin{tabular}{|c|c|}
\hline Definition & Stent Location \\
\hline $\begin{array}{l}\text { Criteria for adequate stent boost }{ }^{11,12} \text { ) } \\
\text { (A stent is optimally deployed if all of following criteria are ful- } \\
\text { filled.) }\end{array}$ & $\begin{array}{l}\text { 1. No sign of focal inadequate expansion, as no protrusion of the stent strut and } \\
\text { no disappearance of continuity of stent struts } \\
\text { 2. Stent minimum diameter }>70 \% \text { of reference diameter } \\
\text { 3. Stent minimum diameter }>2.0 \mathrm{~mm}\end{array}$ \\
\hline Criteria for adequate IVUS ${ }^{13,14)}$ & $\begin{array}{l}\text { 1. MSA at least }>6.5 \mathrm{~mm}^{2} \text { for BMSs and }>5.0 \mathrm{~mm}^{2} \text { for DESs } \\
\text { 2. MSA }>90 \% \text { of the distal reference lumen area or }>80 \% \text { of average reference } \\
\text { lumen area } \\
\text { 3. lack of major dissections, intramural hematomas, and geographic misses }\end{array}$ \\
\hline $\begin{array}{l}\text { Criteria for accuracy of stent location by IVUS and SBS }{ }^{6)} \\
\text { (A stent "misplacement" was defined as the stent being deployed }>1 \\
\text { mm proximal or distal to the true ostium.) }\end{array}$ & $\begin{array}{l}\text { 1. too distal ( }>1 \mathrm{~mm} \text { distal to the IVUS and SBS determined ostium) } \\
\text { 2. too proximal ( }>1 \mathrm{~mm} \text { proximal to he IVUS and SBS determined ostium) } \\
\text { 3. accurate ( }<1 \mathrm{~mm} \text { from the true ostium })\end{array}$ \\
\hline
\end{tabular}




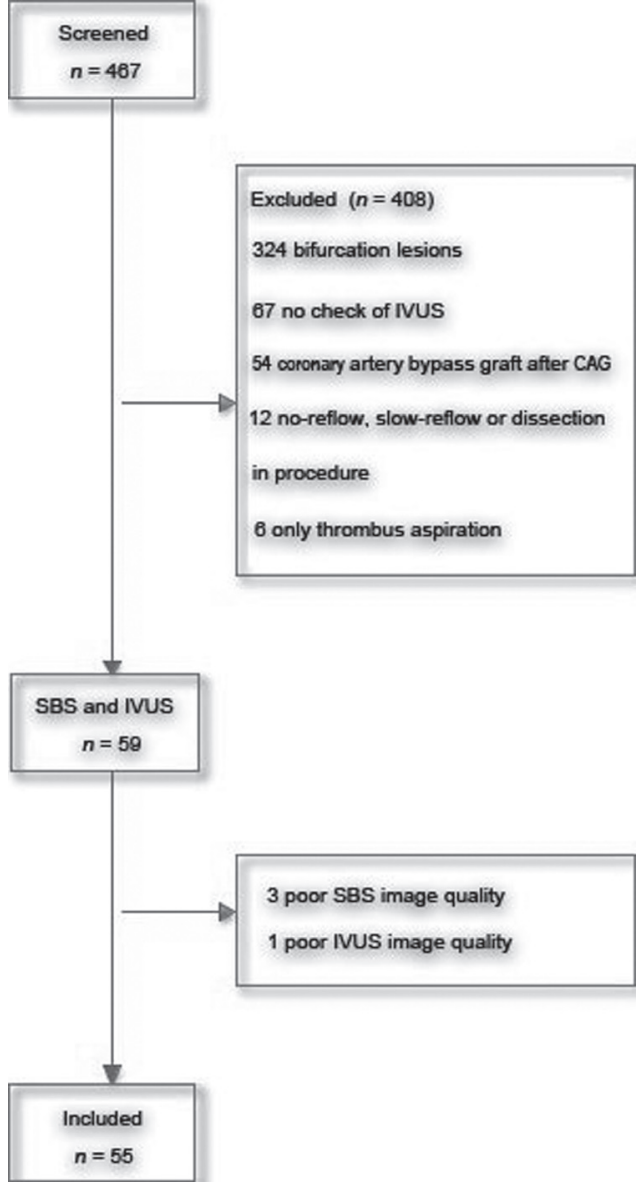

Figure 1. Study flowchart. PCI percutaneous coronary intervention, IVUS intravascular ultrasound, SBS StentBoost Subtract.

tery (RCA). Aorto-ostial lesions $(n=21)$ accounted for $36 \%$ of the lesions, while the remaining $64 \%$ of lesions $(n=37)$ involved major coronary vessels. A single stent was used to treat 53 lesions and 5 lesions were treated using two stents. Drugeluting stents were used in 42 lesions and bare metal stents were used in 16 lesions. The average diameter of the stents was $3.5 \mathrm{~mm}$ and the length was $12.9 \mathrm{~mm}$.

IVUS measurements: Intravascular ultrasound images were obtained immediately after the SBS acquisition in each patient. IVUS revealed abnormal findings in 13 out of 58 observations: inadequate stent expansion in 10 and asymmetrical stent expansion in 3. A decision regarding additional procedures, such as second stenting or high-pressure ballooning, was made on the basis of these IVUS findings.

Stent boost subtract imaging: A total of 58 SBS images were acquired in 58 coronary arteries of 55 patients. After automatic enhancement of the stent edge, quantitative stent measurements were made. The mean reference diameter was $3.5 \pm 0.5$ $\mathrm{mm}$, with a minimum stent diameter of $2.3 \pm 0.5 \mathrm{~mm}$. MSA calculated by minimum stent diameters of two orthogonal directions was $8.3 \pm 1.9 \mathrm{~mm}^{2}$ (Table IV). Eight patients were classified as having inadequate stent expansion by the pre-defined criteria.

Comparison between SBS images and IVUS: A significant positive correlation was observed between SBS-MSA and
Table II. Baseline Clinical Characteristics

\begin{tabular}{lc}
\hline Variable & $n=55$ \\
\hline Age (years) & $65.4 \pm 13.9$ \\
Male & $36(65 \%)$ \\
Medical History & \\
Diabetes mellitus & $19(35 \%)$ \\
Hypertension & $39(71 \%)$ \\
Hyperlipidemia & $45(82 \%)$ \\
Current/former smoker & $34(62 \%)$ \\
Prior percutaneous coronary intervention & $13(24 \%)$ \\
Prior coronary artery bypass graft & $8(15 \%)$ \\
Chronic kidney disease & $4(7 \%)$ \\
Congestive heart failure & $9(16 \%)$ \\
Diagnosis at PCI & \\
ST-elevation myocardial infarction & $3(5 \%)$ \\
Non-ST-elevation myocardial infarction & $11(20 \%)$ \\
Unstable angina pectoris & $34(62 \%)$ \\
Stable angina pectoris & $7(13 \%)$ \\
\hline
\end{tabular}

Table III. Ostial Lesion Characteristics and Procedural Techniques

\begin{tabular}{lc}
\hline Variable & $n=58$ lesions \\
\hline Coronary Ostial Lesion Vessel Location & \\
Aorto-ostial coronary vessel & $21(36 \%)$ \\
$\quad$ Left main & $8(14 \%)$ \\
$\quad$ Right coronary artery & $13(22 \%)$ \\
Major coronary vessel & $37(64 \%)$ \\
$\quad$ Left anterior descending & $24(41 \%)$ \\
Left circumflex & $11(19 \%)$ \\
Ramus intermedius & $2(3 \%)$ \\
Intervention characteristics & \\
Mean number of stents & $1.2 \pm 0.7$ \\
Bare-metal stent(s) & $16(28 \%)$ \\
Drug-eluting stent(s) & $42(72 \%)$ \\
Mean stent length (mm) & $1.29 \pm 6.1$ \\
Mean stent diameter (mm) & $3.5 \pm 0.5$ \\
Rotational atherectomy & $5(9 \%)$ \\
\hline
\end{tabular}

Table IV. Stent Measurements by SBS and IVUS

\begin{tabular}{lr}
\hline Variable & \multicolumn{1}{c}{$n=58$} \\
\hline Stent boost subtract imaging & \\
Minimum diameter $(\mathrm{mm})$ & $2.9 \pm 0.8$ \\
Minimum diameter/reference diameter & $0.79 \pm 0.8$ \\
SBS-MSA $\left(\mathrm{mm}^{2}\right)$ & $8.3 \pm 1.9$ \\
IVUS & \\
IVUS-MSA $\left(\mathrm{mm}^{2}\right)$ & $9.1 \pm 2.3$ \\
Symmetric index & $0.77 \pm 0.9$ \\
\hline
\end{tabular}

MSA by IVUS with a regression coefficient of 0.95 (Figure 2). Correlations of the judgments of the stent results, based on the criteria defined in this study, are shown in Table V. Eight SBS images showed inadequate stent expansion. As a result, SBS predicted inadequate findings of IVUS with $100 \%$ specificity, $80 \%$ sensitivity, $100 \%$ positive predictive value, and $96 \%$ negative predictive value. Meanwhile, a total of 220 images (mean $3.3 \pm 1.5$ ) were obtained and analyzed. The accuracy of stent location was generally good. The location of the stent was accurate in $48(82.8 \%)$, too distal in $6(10.3 \%)$, and too proximal in $4(6.9 \%)$. 


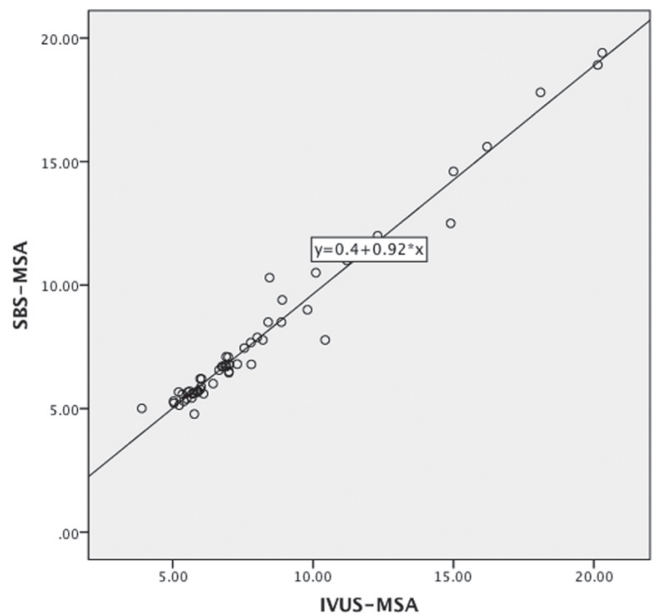

Figure 2. Comparison of the minimum stent area calculated by SBS images and measured by IVUS. There was a significant positive correlation with a regression coefficient of $0.95, P<0.001$.

Table V. Correlations of the Judgments of the Stent Results ( 55 patients, 58 vessels, 58 lesions)

\begin{tabular}{lcc}
\hline & IVUS & \\
& Adequate & Inadequate \\
\hline Stent boost subtract & & \\
Adequate & 48 & 2 \\
Inadequate & 0 & 8 \\
\hline
\end{tabular}

\section{DisCUSSION}

The results of our study suggest that a significant positive correlation was observed between SB-MSA and MSA by IVUS with a regression coefficient of 0.95 , Meanwhile, SBS predicted inadequate findings of IVUS with $100 \%$ specificity and $80 \%$ sensitivity. Furthermore, the accuracy of stent location was generally good, and more specifically, was accurate in $82.8 \%$ of the stents. Our results indicated that SBS images could be used as one of the indices for assessing stent deployment during ostial lesions PCI.

Percutaneous treatment of a coronary artery ostial lesion is a challenging procedure and a matter of constant debate, in which the decision as to the most suitable strategy is not always easy. Percutaneous treatment of ostial lesions is associated with a higher incidence of adverse cardiac events compared with non-ostial lesions. ${ }^{1-4)}$

Imaging techniques play a primary role during ostial lesions PCI and are under constant development. Angiography, considered the standard for the evaluation of coronary lesions, does not permit proper lumen assessment or visualization of stent struts (especially at ostial lesions) after deployment. Intravascular ultrasound (IVUS) is a valuable tool. This technology permits the evaluation of vessel size, lumen diameter (lesion severity), plaque composition, quantification of calcium content, and assessment of complications post intervention. ${ }^{15-17)}$ However, there is a learning curve (requiring trained operators and laboratory staff), and its routine use would significantly increase procedural duration and costs.

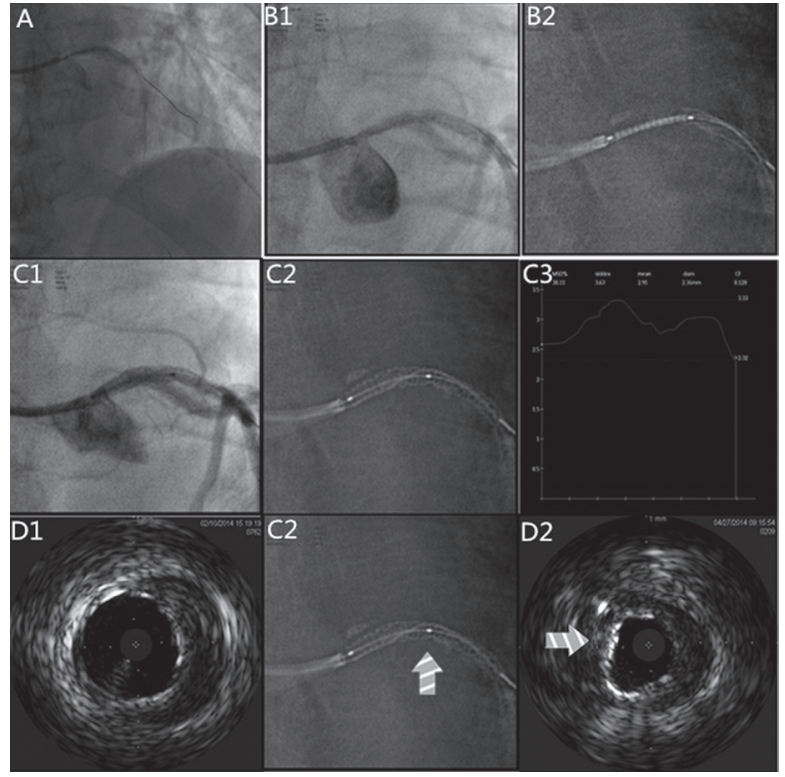

Figure 3. Comparison of SBS imaging and IVUS in a left main lesion. A: Position of stent could not be clearly showed by coronary angiography. B: location of stent was accurately showed between proximal lesions (B1) and distal lesions (B2) before stent was released by SBS imaging, it is especially important for left main ostial lesion. C: Quantitative analysis of the SBS image. SBS images clearly showed location of stent $(\mathbf{C 1}, \mathbf{C 2})$, inadequate stent expansion (C2) and the minimum stent diameter (C3). D: The ostial lesion was totally covered (D1), meanwhile, comparing with inadequate stent expansion by IVUS (D2, arrow), SBS images clearly showed same information (C2, arrow)

Currently, there are many techniques used by interventional cardiologists to approach this subset of ostial lesions. ${ }^{18,19)}$ However, no perfect technique or technologies allow for perfect ostial stent placement in $100 \%$ of cases, and some techniques even have potentially catastrophic failure complications. Moreover, there is definitely a steep learning curve for this technique before it can be applied efficiently in the treatment of all ostial lesions.

Angiographically-guided stenting is the most common and safest technique used for ostial lesion stent placement. However, it was reported that angiographically-guided stenting for coronary ostial disease leads to a high incidence of proximal and distal stent misplacement. ${ }^{6}$ In aorto-ostial lesions in particular, the guiding catheter is often slightly engaged into the target vessel, so angiography could not clearly show the location of the stent (Figure $3 \mathrm{~A}$ ).

SBS is a recently introduced method that permits the visualization of a high quality image of the deployed stent. It does not require the insertion of additional devices in the coronary arteries, does not need specific training of the operator or staff (images are automatically transferred to a workstation and become immediately available in the catheterization laboratory), and does not significantly increase radiation exposure or procedural time ( 3 to 4 -second runs). ${ }^{20)}$ It has been employed more frequently in the evaluation of stent positioning and adequate expansion. ${ }^{21,22)}$ Mishell, et al conducted a study in which they hypothesized that StB use would result in improved identification of underexpanded stent segments relative to standard quantitative angiography (QCA) and IVUS. ${ }^{23)}$ They concluded 
that the 3 techniques had good correlation regarding minimum stent diameter measurements, with a higher correlation between StB and IVUS. Regarding bifurcation PCI, StB has been useful in the evaluation of novel dedicated bifurcation stents. ${ }^{24,25)}$ In the present study, we sought to assess the importance of SBS to guide ostial lesion PCI. Our results indicate that this technique can provide valuable information through several stages of the procedure (balloon and stent positioning, need for post-dilatation) and avoid periprocedural complications, moreover, this information was very important for ostial lesions. Tanaka, et al conducted a study in which they concluded that SB image quantification exhibited rather poor sensitivity $(33 \%),{ }^{26)}$ on the contrary, our results showed good sensitivity $(80 \%)$. The different types of lesions may be one of the important reasons. It might be easy to obtain a clear picture with SBS images due to the lower level of motion artifacts in ostial lesions.

Frequently, angiographic images alone do not permit adequate visualization of stent deformation or incomplete stent expansion at the ostium of a coronary artery. SBS allows for an adequate ostium visualization and is particularly sensitive at detecting severe stent distortion. The visibility of the stent(s) was optimal in most patients. Figure 3 is an example that illustrates the utility of SBS in left main lesions. The use of a standard StB acquisition or a contrast-filled subtraction (SBS) image may clearly show proximal and distal stent location and information about inadequate stent expansion (Figure 3 B1, B2, $\mathrm{C} 1, \mathrm{C} 2$ ), moreover, this result was verified by IVUS (Figure 3 D1, D2). As a result, SBS showed an excellent advantage in terms of inadequate stent expansion and accuracy of stent location, both of which are key during ostial lesion PCI.

Limitations: This is a single-centre study and the sample size was relatively small, thus, these results need to be validated with further studies. SBS has several potential disadvantages: although it results in enhanced image quality, the signal-tonoise ratio is reduced in heavily calcified vessels and segments with multiple stents and, especially in bifurcations, there is the problem of overlap of radiopaque structures (partially solved with a proper view selection). Other potential disadvantages are the inability to describe the lumen and the necessity for additional X-ray exposure to patients and personnel, although the amount of added radiation is small and not likely to be of clinical significance. In addition, this study was not a randomized trial assigning patients to SBS versus non-SBS. Meanwhile, although SBS can only be performed using a Philips system presently, we believe a new tool like SBS will be more extensively applied in other angio systems in the future. We recommend that interventional cardiologists strive to acquire these cheap and quick techniques for accurate ostial stent implantation in the coronary arteries.

Conclusion: Percutaneous treatment of ostial lesions remains a challenging procedure in interventional cardiology and imaging techniques have a primary role to play, permitting proper execution of the selected strategy. SBS is a simple and quick method that offers several advantages, enabling improved stent location, adequate stent expansion, and optimal apposition of the struts to the wall. SBS imaging could be conventionally used during ostial PCI, especially in centers where IVUS is not used routinely.

\section{Disclosure}

Conflict of interest: The authors have no conflicts of interest to declare.

\section{REFERENCES}

1. Lemos PA, Hoye A, Goedhart D, et al. Clinical, angiographic, and procedural predictors of angiographic restenosis after sirolimuseluting stent implantation in complex patients: an evaluation from the Rapamycin-Eluting Stent Evaluated At Rotterdam Cardiology Hospital (RESEARCH) study. Circulation 2004; 109: 1366-70.

2. Mavromatis K, Ghazzal Z, Veledar E, et al. Comparison of outcomes of percutaneous coronary intervention of ostial versus nonostial narrowing of the major epicardial coronary arteries. Am J Cardiol 2004; 94: 583-7.

3. Freeman M, Clark DJ, Andrianopoulos N, et al. Outcomes after percutaneous coronary intervention of ostial lesions inthe era of drug-eluting stents. Catheter Cardiovasc Interv 2009; 73: 763-8.

4. Hur SH, Kang SJ, Kim YH, et al. Impact of intravascular ultrasound-guided percutaneous coronary intervention on long-term clinical outcomes in a real world population. Catheter Cardiovasc Interv 2013; 81: 407-16.

5. Jokhi P, Curzen N. Percutaneous coronary intervention of ostial lesions. Eurointervention 2009; 5: 511-4.

6. Dishmon DA, Elhaddi A, Packard K, Gupta V, Fischell TA. High incidence of inaccurate stent placement in the treatment of coronary aorto-ostial disease. J Invasive Cardiol 2011; 23: 322-6.

7. Puri R, Kapadia SR, Nicholls SJ, Harvey JE, Kataoka Y, Tuzcu EM. Optimizing outcomes during left main percutaneous coronary intervention with intravascular ultrasound and fractional flow reserve: the current state of evidence. JACC Cardiovasc Interv 2012; 5: 697-707. (Review)

8. Sonoda S, Morino Y, Ako J, et al. Impact of final stent dimensions on long-term results following Sirolimus-eluting stent implantation serial intravascular ultrasound analysis from the sirius trial. J Am Coll Cardiol 2004; 43: 1959-63.

9. Fujii K, Carlier SG, Mintz GS, et al. Stent under expansion and residual reference segment stenosis are related to stent thrombosis after sirolimus-eluting stent implantation: an intravascular ultrasound study. J Am Coll Cardiol 2005; 45: 995-8.

10. Cook S, Wenaweser P, Togni M, et al. Incomplete stent apposition and very late stent thrombosis after drug-eluting stent implantation. Circulation 2007; 115: 2426-34.

11. Orford JL, Lerman A, Holmes DR. Routine intravascular ultrasound guidance of percutaneous coronary intervention: a critical reappraisal. J Am Coll Cardiol 2004; 43: 1335-42. (Review)

12. Koolen JJ, van het Veer M, Hanekamp CE. StentBoost image enhancement: first clinical experience. Medicamundi 2005; 49: 4-8.

13. Park SJ, Kim YH, Park DW, et al. Impact of intravascular ultrasound guidance on long-term mortality in stenting for unprotected left main coronary artery stenosis. Circ Cardiovasc Interv 2009; 2: 167-77.

14. Jakabcin J, Spacek R, Bystron M, et al. Long-term health outcome and mortality evaluation after invasive coronary treatment using drug eluting stents with or without the IVUS guidance. Randomized control trial. HOME DES IVUS. Catheter Cardiovasc Interv 2010; 75: 578-83.

15. Chieffo A, Park SJ, Valgimigli M, et al. Favorable long-term outcome after drug-eluting stent implantation in nonbifurcation lesions that involve unprotected left main coronary artery: a multicenter registry. Circulation 2007; 116: 158-62.

16. Al-Lamee R, Ielasi A, Latib A, et al. Comparison of long-term clinical and angiographic outcomes following implantation of bare metal stents and drug-eluting stents in aorto-ostial lesions. Am J Cardiol 2011; 108: 1055-60.

17. Levine GN, Bates ER, Blankenship JC, et al. 2011 ACCF/AHA/ SCAI Guideline for Percutaneous Coronary Intervention. A report 
of the American College of Cardiology Foundation/American Heart Association Task Force on Practice Guidelines and the Society for Cardiovascular Angiography and Interventions. J Am Coll Cardiol 2011; 58: e44-122.

18. Fischell TA, Saltiel FS, Foster MT, Wong SC, Dishman DA, Moses J. Initial clinical experience using an ostial stent positioning system (Ostial Pro) for the accurate placement of stents in the treatment of coronary aorto-ostial lesions. J Invasive Cardiol 2009; 21: 53-9.

19. Kern MJ, Ouellette D, Frianeza T. A new technique to anchor stents for exact placement in ostial stenoses: the stent tail wire or Szabo technique. Catheter Cardiovasc Interv 2006; 68: 901-6.

20. Jin Z, Yang S, Jing L, Liu H. Impact of StentBoost subtract imaging on patient radiation exposure during percutaneous coronary intervention. Int J Cardiovasc Imaging 2013; 29: 1207-13.

21. Okabe T, Kawamura A, Maekawa Y, et al. Can intravascular ultrasound guidance modify the efficacy of drug-eluting stent over bare-metal stent in an aorto-ostial lesion? Cardiovasc Revasc Med 2011; 12: 105-10.
22. Hassan AK, Bergheanu SC, Stijnen T, et al. Late stent malapposition risk is higher after drug-eluting stent compared with baremetal stent implantation and associates with late stent thrombosis. Eur Heart J 2010; 31: 1172-80. (Review)

23. Kang SJ, Mintz GS, Park DW, et al. Mechanisms of in-stent restenosis after drug-eluting stent implantation: intravascular ultrasound analysis. Circ Cardiovasc Interv 2011; 4: 9-14.

24. Agostoni P, Verheye S. Novel self-expanding stent system for enhanced provisional bifurcation stenting: Examination by StentBoost and intravascular ultrasound. Catheter Cardiovasc Interv 2009; 73: 481-7.

25. Agostoni P, Verheye S. Bifurcation stenting with a dedicated biolimus-eluting stent: X-ray visual enhancement of the final angiographic result with "StentBoost Subtract". Catheter Cardiovasc Interv 2007; 70: 233-6.

26. Tanaka N, Pijls NH, Koolen JJ, et al. Assessment of optimum stent deployment by stent boost imaging: comparison with intravascular ultrasound. Heart Vessels 2013; 28: 1-6. 